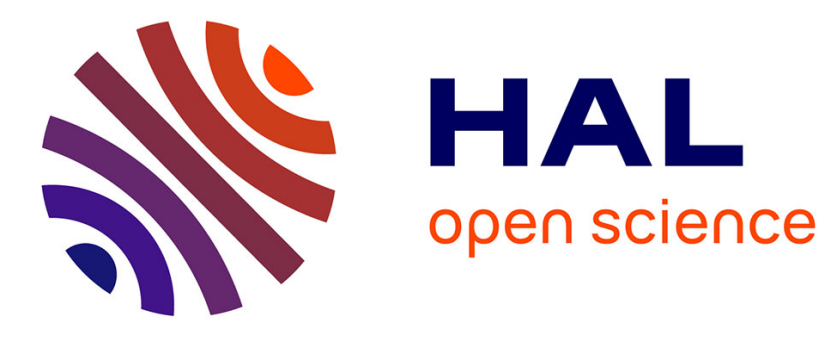

\title{
Modeling of IEEE 802.11 Multi-hop Wireless Chains with Hidden Nodes
}

Thiago Abreu, Bruno Baynat, Thomas Begin, Isabelle Guérin-Lassous, Nghi Nguyen

\section{- To cite this version:}

Thiago Abreu, Bruno Baynat, Thomas Begin, Isabelle Guérin-Lassous, Nghi Nguyen. Modeling of IEEE 802.11 Multi-hop Wireless Chains with Hidden Nodes. 17th ACM International Conference on Modeling, Analysis and Simulation of Wireless and Mobile Systems (2014), Sep 2014, Montréal, Canada. pp.159-162, 10.1145/2641798.2641826 . hal-01071825

\section{HAL Id: hal-01071825 \\ https://hal.science/hal-01071825}

Submitted on 6 Oct 2014

HAL is a multi-disciplinary open access archive for the deposit and dissemination of scientific research documents, whether they are published or not. The documents may come from teaching and research institutions in France or abroad, or from public or private research centers.
L'archive ouverte pluridisciplinaire HAL, est destinée au dépôt et à la diffusion de documents scientifiques de niveau recherche, publiés ou non, émanant des établissements d'enseignement et de recherche français ou étrangers, des laboratoires publics ou privés. 


\section{Modeling of IEEE 802.11 Multi-hop Wireless Chains with Hidden Nodes}

\author{
Thiago Abreu \\ Université Lyon 1 - LIP \\ France \\ thiago.wanderley@ens-lyon.fr \\ Isabelle Guérin-Lassous \\ Université Lyon 1 - LIP \\ France \\ isabelle.guerin-lassous@ens- \\ lyon.fr
}

\author{
Bruno Baynat \\ Université Pierre et Marie \\ Curie - LIP6 \\ France \\ bruno.baynat@lip6.fr
}

\author{
Thomas Begin \\ Université Lyon 1 - LIP \\ France \\ thomas.begin@ens-lyon.fr
}

\author{
Nghi Nguyen \\ Université Lyon 1 - LIP \\ France \\ huu.nguyen@ens-lyon.fr
}

\begin{abstract}
In this paper, we follow up an existing modeling framework to analytically evaluate the performance of multi-hop flows along a wireless chain of four nodes. The proposed model accounts for a non-perfect physical layer, handles the hidden node problem, and is applicable under workload conditions ranging from flow(s) with low intensity to flow(s) causing the network to saturate. Its solution is easily and quickly obtained and delivers estimates for the expected throughput and for the datagram loss probability of the chain with a good accuracy.
\end{abstract}

\section{INTRODUCTION}

Most WLANs are based on the IEEE 802.11 standard, which implements a probabilistic media access control (MAC) layer. The IEEE 802.11 standard is generally appreciated for its ease of implementation and simple configuration. However, the performance evaluation of these WLANs are generally not straightforward because of their non-deterministic nature (due to the use of a probabilistic contention algorithm and to the dynamic behavior of the radio medium).

In the case of infrastructure mode, where nodes communicate through an access point, a large body of analytical models have been proposed in the literature $[3,6]$. These models afford a quick means for researchers and practitioners to forecast many aspects of a WLAN behavior before its deployment, or to better set it up.

Multi-hop wireless networks are another type of WLANs where each node participates in routing by forwarding packets for other nodes. Typically, their decentralized nature makes them suitable for cases where there are no central nodes or for emergency situations like natural disasters. How-

Permission to make digital or hard copies of all or part of this work for personal or classroom use is granted without fee provided that copies are not made or distributed for profit or commercial advantage and that copies bear this notice and the full citation on the first page. Copyrights for components of this work owned by others than the author(s) must be honored. Abstracting with credit is permitted. To copy otherwise, or republish, to post on servers or to redistribute to lists, requires prior specific permission and/or a fee. Request permissions from permissions@ acm.org. MSWiM'14, September 21-26, 2014, Montreal, QC, Canada.

Copyright is held by the owner/author(s). Publication rights licensed to ACM. ACM 978-1-4503-3030-5/14/09 ...\$15.00.

http://dx.doi.org/10.1145/2641798.2641826. ever, multi-hop wireless networks raise new issues with regards to the routing protocols and to the discovery and refinement of their performance.

This paper addresses the performance evaluation of a multihop wireless network based on IEEE 802.11, where packets need to hop several relay nodes before reaching their final destination. We refer to these networks as chains. There is only a handful of works specifically devoted to the analytical performance evaluation of such chains based on IEEE 802.11 $[2,4,1]$. Besides, it seems that none of them handles at the same time realistic assumptions regarding the behavior of the MAC protocol, the inter-dependencies in the distribution of the workload among the nodes (some nodes may be in saturation while others may be in starvation) and the hidden node problem, which are fundamental properties of a wireless chain with several nodes.

In a previous paper [1], we analyzed the behavior of the simplest chain which has only one relay node. Though a necessary milestone, its limited size allowed us to overlook the well-known but complex issue of hidden node problem. The contributions of this paper are twofold. First, we extend our modeling framework to evaluate the performance for a flow conveyed through larger chains in which the hidden node problem takes place. The solution to the model is based on a simple iterative scheme that is solved typically within less than a second. In general, the proposed model delivers good forecasts for the expected throughput and for the datagram losses as a function of the actual positions of the relay nodes and for various values of the flow rate. Second, our model affords a convenient means to quickly investigate the performance behavior of a chain under various conditions. Given the fast solution of our model, we explore many possible configurations for the relay nodes and for the levels of the flow rate in order to get a better understanding of multi-hop wireless networks and to highlight properties inherent to those networks.

\section{SCENARIO DESCRIPTION}

The scenario under consideration is depicted in Figure 1. It consists of a wireless multi-hop chain with 4 nodes, each equipped with a single IEEE 802.11 communication interface. The nodes communicate using the DCF mode of IEEE 


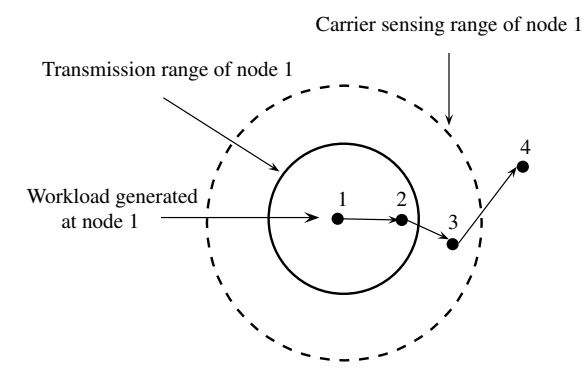

Figure 1: Multi-hop chain with 4 nodes

802.11. In our scenario, we disabled the RTS/CTS mechanism since it is known to be inefficient in the case of a chain [7]. Every node can communicate only with its 1hop neighbors, but its carrier sensing range covers its 2-hop neighbor nodes. Note that there are no restrictions to the nodes alignment and position, as long as they meet the aforementioned assumptions for the communication and for the carrier sensing ranges.

The physical layer used for the frame transmission is unreliable (non-perfect), and therefore, frames may be lost because of bits error or alteration. This is taken into account by the Bit Error Rate (BER), which gives the probability that a bit is misinterpreted at a receiver node due to the transmission process (which includes noise, distortion, attenuation, etc). In our study, the BER is affected by the propagation process and noise. In addition to BER, frames may also be lost when nearby nodes are transmitting simultaneously, which causes frame collisions. In a four-nodes chain, collisions are frequent since nodes 1 and 4 are exposed to the hidden node problem.

The four-nodes chain conveys packets (datagrams) from node 1 up to node 4 (see Figure 1). All datagrams are of same length and the datagrams generation at node 1 follows a Poisson process with a rate $\lambda_{1}$. This flow of datagrams constitutes the workload for the chain.

\section{MODEL}

In [1] we introduce the first parts of a general framework for performance evaluation of a multi-hop wireless chain. The analysis was restricted to a simple scenario with only three nodes. Here, we extend markedly our proposed framework by introducing one important missing feature in the previous analysis, i.e., the hidden node problem which takes place in larger chains. Unlike our previous study of [1] in which virtually no frames collisions can occur, larger chains are prone to frequent frames collisions. In this paper, we present a practical means to handle these collisions within our modeling framework. By doing so, we also demonstrate that our preliminary work is extendable to more general scenarios, with the ultimate objective being to derive a whole framework for the performance evaluation of any multi-hop wireless chain.

Following the framework developed in [1], our model is composed of two levels: a global queueing network model, and several local Markov chain models. Since only three nodes are effectively transmitting frames (node 4 only returns acknowledgments), the global queueing model associated with the chain is composed of three queues with fi-

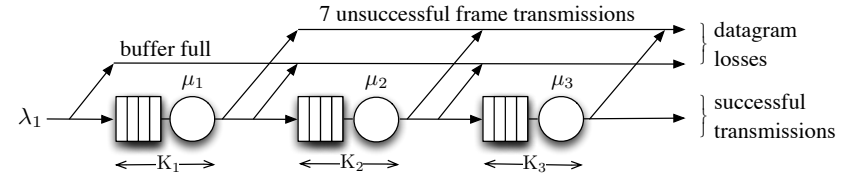

Figure 2: Global queueing model

nite buffer as illustrated in Figure 2. The customers of this queueing model are the datagrams of the chain and the buffer size of queue $i$ is denoted by $K_{i}$. The service rate $\mu_{i}$ of queue $i$ is, by definition, the inverse of the mean service time $S_{i}$ of queue $i$, which corresponds to the average time node $i$ needs to transmit a datagram that is ready to be sent over the radio channel. As developed in [1], $S_{i}$ includes all successive frame (re)transmissions (corresponding to the considered datagram), as well as all IEEE 802.11 DCF protocol delays (DIFS, backoff, SIFS, timeout) and all freezing times due to another node transmission during the backoff of node $i$. This parameter will be estimated thanks to the local Markovian model associated with node $i$. The local Markov chain models are similar to those presented in [1].

Like in our previous model [1], a datagram can be lost either because of a buffer overflow or because of excessive retransmissions of the associated frames. But there is a fundamental difference between the global model presented here and the one previously developed in [1]: a frame loss can either be due to low quality of the channel or to collision over the shared medium. The frame loss probability $p_{f_{i}}$ of node $i$ must account for these two possibilities, whereas in [1] it was only related to the BER. If we denote by $p_{B E R_{i}}$ the probability that a given frame sent by node $i$ is lost because of the BER, and by $p_{\text {coll }_{i}}$ the probability that the frame sent by node $i$ is lost because of a collision with another frame, and if we assume that these two events are independent but not disjoint (a frame can be both in error and in collision), the frame loss probability $p_{f_{i}}$ of node $i$ can be obtained as:

$$
p_{f_{i}}=p_{\text {coll }_{i}}+p_{B E R_{i}}-p_{\text {coll }_{i}} p_{B E R_{i}}
$$

As for the BER probability, which was solely used in [1] for estimating the frame error probability, its derivation remains identical. However, the collision probability, which was virtually null in the case handled by [1], can not be neglected and, as will be seen later, is a very sensitive parameter that must be carefully estimated.

A frame collision may be the result of two different factors. First, a collision can result from the well known hidden problem of two nodes that are not in the carrier sensing range of each other. Second, because of the sensing mechanism of 802.11, a collision can also occur when two neighboring nodes finish their backoff countdown simultaneously. By assuming that these two possibilities result in disjoint events (which turns out to be exact in our scenario), we can decompose the frame collision probability of node $i$ as the sum of the probability of both events:

$$
p_{\text {coll }_{i}}=p_{\text {hid }_{i}}+p_{\text {st }_{i}}
$$

Let us first consider the hidden problem case and see how we can estimate the collision probability at node $i$ due to frame collision with nodes that are hidden from node $i$, denoted as $p_{h i d_{i}}$. In our scenario, since we assume a 2-hop carrier sensing range, the hidden problem can only take place 


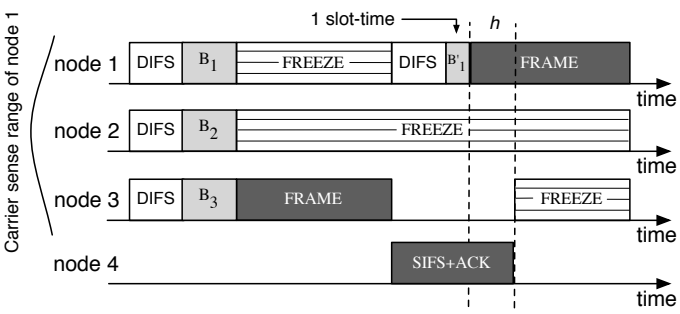

Figure 3: Collision between ACK from node 4 and frame from node 1

between node 1 and node 4, and more precisely, between a data frame sent by node 1 to node 2 and an acknowledgement ("ACK") sent back by node 4 to node 3 . As an illustration, in Figure 3, node 3 senses the medium idle for the duration of its backoff and then starts the transmission of a frame, freezing the backoff countdowns of nodes 1 and 2. The associated acknowledgement ("ACK") sent by node 4 does not prevent node 1 from resuming its backoff countdown (event " $B_{1}^{\prime}$ "), since nodes 1 and 4 are hidden. If the remaining backoff of node 1 is short enough (it corresponds to 1 time-slot in the example), node 1 will transmit its frame and a collision will occur for both frame and ACK. Although the ACK from node 4 collides, we consider that a collision happens at node 3 , since the retransmission mechanism will be performed by this node.

As can be seen on the figure, the duration of the collision is bounded by the maximum overlap $h$ between the frame transmission of node 1 and the ACK transmission of node 4 :

$$
h=\mathrm{SIFS}+\mathrm{ACK}-\mathrm{DIFS}-1 \text { time slot }
$$

We subtract 1 time slot to it, since after a backoff freezing period, the remaining backoff has at least 1 time slot to decrement.

By considering that the nodes have always a frame to transmit, the collision probability $p_{h_{i d}}$ of node $i$ due to a hidden node $j$ can be estimated in a first approximation as the ratio between the duration of a possible collision $(h)$ and the time during which a collision may take place $\left(h+\bar{B}_{j}\right)$ in between two transmissions of node $i$ :

$$
p_{h_{i d}}=\frac{h}{h+\bar{B}_{j}}
$$

In this relation, $\bar{B}_{j}$ is the mean backoff duration of node $j$ (see [1] for more details).

Relation (4) has however two limitations. First, by only considering the average duration of the backoff $\left(\overline{B_{j}}\right)$, we do not take into account the high variability of the binary exponential backoff used in IEEE 802.11. For instance, if node 1 is in the first stage of its backoff, an ACK from node 4 will very likely collide with a frame of node 1 . Conversely, when node 1 is in one of the last stages of its backoff, an ACK from node 4 will have a high chance to be transmitted successfully. Let us define $t b_{j}(k)$, the proportion of time during which hidden node $j$ remains in backoff stage $k . t b_{j}(k)$ is the ratio between the average time effectively spent in the $k$-th backoff stage of node $j\left(p_{f}^{k-1} t_{k, j}\right)$ and the average service time of node $j\left(S_{j}\right)$ :

$$
t b_{j}(k)=\frac{p_{f_{j}^{k-1}}^{k-1} t_{k, j}}{S_{j}}
$$

The collision probability $p_{h i d_{i}}$ can thus be rewritten as:

$$
p_{\text {hid } i}=\sum_{k=1}^{7} t b_{j}(k) \frac{h}{h+\bar{B}(k)}
$$

where $\bar{B}(k)$ is the average backoff duration at stage $k$.

Second, relation (4) (or equivalently relation (6)) implicitly assumes that node $j$ has a datagram to transit (otherwise no collision can occur with node $i$ ) and should actually

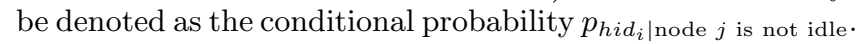
From the law of Total Probabilities, we can obtain the unconditioned collision probability, by noting that the probability $p_{\text {hid }_{i} \mid \text { node }} j$ is idle is null and by reminding that the probability that node $j$ is not idle is nothing but node $j$ utilization (denoted $\bar{U}_{j}$ ):

$$
p_{\text {hid }_{i}}=p_{\text {hid }_{i} \mid \text { node }} j \text { is not idle } \bar{U}_{j}
$$

By combining previous relations, the collision probability due to hidden nodes can finally be expressed as:

$$
p_{\text {hid }_{i}}=\sum_{k=1}^{7} t b_{j}(k) \frac{h}{h+\bar{B}(k)} \bar{U}_{j}
$$

Let us now consider the possible simultaneous transmissions of two neighboring nodes. As explained above, two nodes in the carrier sensing range of each other are very likely to synchronize themselves (mainly when the load is high). And there is a non negligible probability that the backoff countdowns of these two nodes expire simultaneously and that the two nodes start their transmission exactly at the same time, resulting in frame collisions. Let $p_{s t_{i}}$ denote the probability that a frame of node $i$ collides with a frame of any node that is in its carrier sensing range and that starts a transmission at the same time as node $i$. This probability can be estimated as follows:

$$
p_{s t_{i}}=1-\prod_{j \neq i}\left(1-\tau_{j} \bar{U}_{j}\right)
$$

where $\tau_{j}$ is the probability that a given node $j$ in the carrier sensing range of node $i$ starts its transmission at the same time as node $i$, provided node $j$ has something to transmit. This is an approximation, assuming that three (or more) nodes have a very small chance to start their transmission all together. Now we simply estimate the missing conditional probability $\tau_{j}$ as the inverse of the average backoff duration of node $j$ expressed in number of time slots:

$$
\tau_{j}=\frac{1}{\bar{B}_{j}}
$$

\section{NUMERICAL RESULTS}

In this section we address the accuracy concern of our proposed model. Throughout this section, we use the parameter values of IEEE $802.11 \mathrm{~b}$ as reported in Table 1 . The communication and carrier sensing ranges cover 399 and 700 meters, respectively. The received signal power at each node is computed using a transmission power of $31.6 \mathrm{~mW}$, an antenna gain of $1 \mathrm{dBi}$ and the two-ray ground reflection model. We derive the BER, which accounts for the non-perfect physical layer, based on a relation between the received signal power and the used modulation.

To evaluate the accuracy of our model, we compare its performance parameters obtained with an implementation 


\begin{tabular}{|c|c|}
\hline DIFS & $50 \mu \mathrm{s}$ \\
\hline SIFS & $10 \mu \mathrm{s}$ \\
\hline Time slot & $20 \mu \mathrm{s}$ \\
\hline Contention window size (min,max) & 31,1023 \\
\hline Frame retransmission limit & 7 \\
\hline Physical rate & $11 \mathrm{Mb} / \mathrm{s}$ \\
\hline
\end{tabular}

Table 1: IEEE 802.11b parameters

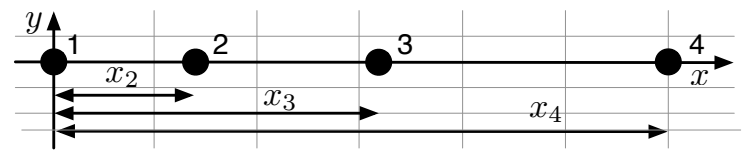

Figure 4: Topology used for the numerical results

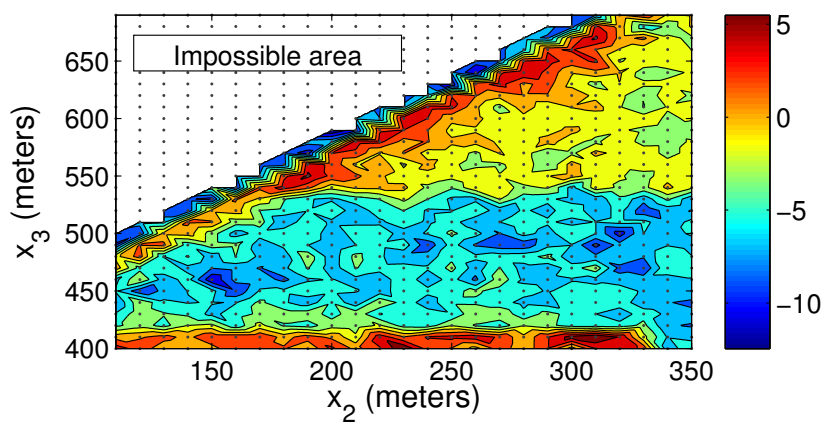

Figure 5: Percentage relative errors for the expected throughput for various positions of relay nodes $\left(x_{2}\right.$ and $\left.x_{3}\right)$ with $K_{i}=20$ and $\lambda_{1}=2 \mathrm{Mb} / \mathrm{s}$

in MATLAB with those delivered by a discrete-event simulator (Network Simulator version 2.35 - ns-2.35 [5]) for a large set of possible chains with four nodes. Note that all simulation results have been performed by generating 100,000 packets at the source node. We define the percentage relative error of our model versus the actual values (delivered by ns-2.35) as the ratio $100 \times$ (approximate - actual) / actual. To simplify the presentation of the results, the four nodes of the chain are scattered in a straight line as illustrated by Figure 4 . Nodes 1 and 4 are steady while the positions of nodes 2 and 3 vary. We denote by $x_{i}$ the distance between node i and node 1 . Values of $x_{2}$ are within the interval [110, 350] meters, while $x_{3}$ belongs to the interval [400, 690] meters. $x_{4}$ is constantly set to 750 meters. Note that the positions of nodes 2 and 3 must obey certain rules so that 1hop neighbors can communicate. This is the reason behind the white "impossible area" band in Figure 5. Aside from $x_{i}$, two additional parameters can be tuned in our scenario: the size of buffers $K_{i}$, and the workload rate $\lambda_{1}$. Datagrams have a size of 1500 bytes.

We focus on the ability of our model to provide fair predictions for the expected throughput of the 4-nodes chain. Figure 5 represents the relative error value on the chain throughput for our proposed model as a function of the distances of both relay nodes 2 and 3 to node 1 .
In this example, the buffers are of length $K_{i}=20$, and the workload is set to a high, but not excessive level, which corresponds to a datagrams arrivals rate at the source node (node 1) of $\lambda_{1}=2 \mathrm{Mb} / \mathrm{s}$. We choose this value of $\lambda_{1}$ because it leads to analytical difficulties as the buffer at node 1 is neither completely full nor empty. Note that this figure corresponds to hundreds of data points explored (both by the simulator and by the model), and the surfaces shown are obtained using an interpolation from sets of scattered data points. The relative error tends to be low as it stands below $10 \%$ for virtually all of the nearly 550 configurations we have performed to generate this figure. We observe that the relative error tends to attain its maximum value (near $10 \%$ ) (in the top points of the figure) when the link between nodes 2 and 3 is at is maximum distance, causing very high values of BER and a frame loss probability exceeding $60 \%$. By studying the overall distribution of relative errors in the throughput, we observe that the mean error is around $4 \%$, in close to $90 \%$ of cases the error remains below $10 \%$ and it never exceeds $15 \%$ in all considered cases.

We have assessed the accuracy of our model on other performance parameters, e.g. the datagram loss probability, as well as on many different configurations. It is our conclusion that, in general, the accuracy of the model is good and that the results presented above reflect its typical behavior.

\section{Acknowledgments}

This work was funded by the French National Research Agency (ANR) under the projects ANR VERSO RESCUE (ANR-10-VERS-003) and ANR-JST PETAFLOW (ANR09-BLAN-0376).

\section{REFERENCES}

[1] T. Abreu, B. Baynat, T. Begin, and I. Guérin-Lassous. Hierarchical Modeling of IEEE 802.11 Multi-hop Wireless Networks. In Proceedings of ACM MSWiM, pages 143-150. ACM, 2013.

[2] A. Aziz, M. Durvy, O. Dousse, and P. Thiran. Models of 802.11 multi-hop networks: Theoretical insights and experimental validation. In IEEE COMSNETS, 2011.

[3] G. Bianchi. Performance analysis of the IEEE 802.11 distributed coordination function. IEEE JSAC, 18(3), 2000.

[4] M. M. Hira, F. A. Tobagi, and K. Medepalli. Throughput analysis of a path in an IEEE 802.11 multihop wireless network. In Proceedings of IEEE WCNC, 2007.

[5] NS2. http://www.isi.edu/nsnam/ns/.

[6] I. Tinnirello, G. Bianchi, and Y. Xiao. Refinements on IEEE 802.11 distributed coordination function modeling approaches. IEEE Transactions on Vehicular Technology, 59(3), 2010.

[7] K. Xu, M. Gerla, and S. Bae. Effectiveness of RTS/CTS handshake in IEEE 802.11 based ad hoc networks. Ad Hoc Networks, 1, 2003. 